\title{
Tagungsforum
}

\section{Zehn Jahre Schlüsselqualifikationen im Deutschen Richtergesetz}

\section{- Symposium an der Universität Regensburg (Ausbildungszentrum REGINA) - \\ Johannes Weber"}

Am 1.7.2003 trat das Gesetz zur Reform der Juristenausbildung in Kraft. Seither ist

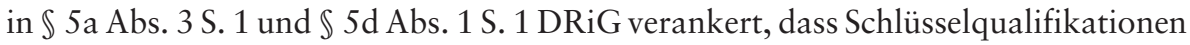
im Jurastudium und in Prüfungen zu berücksichtigen sind. Diesem Ausbildungsauftrag widmete sich am 21. und 22.11.2013 ein Symposium, zu dem das Ausbildungszentrum REGINA an die Universität Regensburg bundesweit eingeladen hatte. $\mathrm{Zu}$ der interdisziplinären Arbeitstagung trafen sich Professoren ${ }^{1}$, Mittelbauvertreter, Studenten sowie Vertreter juristischer Berufsverbände. Leitgedanke dabei war, das Jurastudium im Bereich der Schlüsselqualifikationen weiter zu verbessern. Das Symposium gliederte sich in drei Phasen: ${ }^{2}$

- Grundsatzphase, in der die unterschiedlichen Sichtweisen zu juristischen Schlüsselqualifikationen dargelegt wurden.

- Arbeitsphase, in der Erfahrungen und Angebote in vier Workshops zunächst reflektiert und diskutiert wurden. In einem zweiten Schritt fassten die Teilnehmenden die Diskussionsergebnisse und Empfehlungen in konkrete Thesen.

- Ergebnisphase, in der die erarbeiteten Thesen vorgestellt und vom Podium und dem Plenum diskutiert und letztlich abgestimmt wurden.

Als Rahmenprogramm bot das Symposium am ersten Abend einen Redewettstreit unter Jurastudenten mit über 400 Besuchern. Am zweiten Abend hielt der Hirnforscher Manfred Spitzer den öffentlichen Festvortrag „Gehirnforschung für Juristen“.

\section{A. Der Wert von Schlüsselqualifikationen}

Zum Auftakt des Symposiums umrissen sechs Grundsatzreferate die Bedeutung von Schlüsselqualifikationen aus interdisziplinärer, berufspraktischer, universitärer und studentischer Perspektive:

\section{Schlüsselqualifikationen funktionieren ohne Inhalt nie}

Aus interdisziplinärer Sicht ging Michael Thiele (Universität Frankfurt a.M.) der Frage „Wozu Schlüsselqualifikationen?" nach. Er definierte Schlüsselqualifikationen

* Der Autor ist wissenschaftlicher Mitarbeiter am Ausbildungszentrum REGINA der Universität Regensburg; unterstützt durch Vanessa Jäger und Jens Bruns.

1 Im folgenden Text wird zur besseren Lesbarkeit das generische Maskulinum verwendet. Implizit sind damit alle Geschlechter diskriminierungsfrei angesprochen.

2 Detailliertes Programm unter http://www.uni-regensburg.de/rechtswissenschaft/fakultaet/regina/sq/index.html (8.4.2014). 
beispielhaft über Kommunikationsfähigkeit, Präsenz, Gesprächsführung, Fingerspitzengefühl, Redetalent, Soziale Kompetenz und Personalmanagement. In den Mittelpunkt seiner Ausführungen stellte er die Rhetorik. An diesem Beispiel zeigte Thiele u. a., dass Schlüsselqualifikationen ohne Inhalt nie funktionieren.

\section{Im juristischen Berufsalltag haben Schlüsselqualifikationen eine bedeutende Rolle}

Für den Richterberuf führte Lore Sprickmann-Kerkerinck (Deutscher Richterbund) erforderliche Fertigkeiten im Bereich der Kommunikation, Gesprächsführung, Vernehmungslehre und Streitschlichtung auf. Sie forderte ein entsprechendes Lehrangebot an den Universitäten, wobei sie Persönlichkeitskompetenzen während des Studiums für nur bedingt erlernbar hielt.

Auch für die anwaltschaftlichen Kernaufgaben sind Schlüsselqualifikationen zwingend erforderlich. Hansjörg Staeble (Bundesrechtsanwaltskammer) warf dabei aber die Frage auf, ob die Universität für die Vermittlung der geeignete Ort sei oder ob nicht ein „learning on the job“ (so: Hans-Jürgen Rabe, Deutscher Anwaltverein) ausreiche. Da aber Grundkenntnisse bereits vor Berufsbeginn essentiell seien, forderte Staehle schon im Jurastudium eine Vermittlung konkreter Grundkenntnisse von Schlüsselqualifikationen wie Rhetorik, Vernehmungslehre und Mediation.

\section{Die Universitäten betrachten die Vermittlung von Schlüsselqualifikationen kontrovers}

Christian Wolf (Universität Hannover; „Schlüsselqualifikationen - ein Irrtum“) sieht in den zusätzlichen universitären Schlüsselqualifikationsangeboten eine weitere „Trivialisierung der Universität“. Lediglich Moot Courts sowie das erste traditionelle Seminar seien sinnvolle Vermittlungsformen von Schlüsselqualifikationen.

Die Schlüsselqualifikationen als Irrtum zu sehen, beschrieb Hendrik Schneider (Universität Leipzig; „Schlüsselqualifikationen - ein Erfolg“) dagegen als anachronistisch. Er sprach sich für die Einbindung der Schlüsselqualifikationen in die universitäre Ausbildung aus, da das Studium auch für den Arbeitsmarkt befähigen müsse.

\section{Studenten begrüßen Schlüsselqualifikationen, bemängeln aber die Umsetzung}

Julia Hörnig (Bundesverband rechtswissenschaftlicher Fachschaften) kritisierte den status quo des Veranstaltungsangebots und forderte Verbesserungen bezüglich der Einheitlichkeit des Kursangebots und der Qualität der Veranstaltungen. Prinzipiell seien Schlüsselqualifikationskurse wichtig und sollten vor allem als Debattier- und Rhetorikkurse oder Moot Courts ausgestaltet werden. Dabei könne noch stärker auf die Erfahrung von Praktikern zurückgegriffen werden. 


\section{B. Ergebnisse des Symposiums}

Die wichtigsten Ergebnisse der Workshops, der Podiumsdiskussion und der abschließenden Plenumsabstimmung sind:

\section{I. „Schlüsselqualifikationen“ i.S.v. §5a Abs. 3 S. 1 DRiG werden zu weit ausgelegt}

Der Gesetzgeber umschreibt in $\mathbb{\int} 5$ a Abs. 3 S. 1 DRiG den Begriff „Schlüsselqualifikationen " anhand einer beispielhaften ${ }^{3}$ Aufzählung. So entstand für die Landesgesetzgeber ${ }^{4}$ und die Fakultäten ein großer Gestaltungsspielraum, was unter den Begriff „Schlüsselqualifikationen“ zu fassen ist. In der Folge haben die Fakultäten in den letzten Jahren neben den sieben „klassischen“ (in $\mathbb{\$} 5$ a Abs. 3 S. 1 DRiG aufgeführten) Schlüsselqualifikationen noch ca. 260 weitere Angebote („neue“ Schlüsselqualifikationen) in die Lehre aufgenommen. Die Bandbreite der „neuen“ Schlüsselqualifikationen reicht von BWL-Grundlagen über PR-Arbeit im Kulturbereich bis hin zu Computerkursen. Das Symposium kritisiert deshalb, dass der Begriff „Schlüsselqualifikationen“ häufig zu weit ausgelegt wird. Da die im Gesetz aufgezählten Beispiele allesamt einen kommunikativen Kern beinhalten, sollen entsprechend der ratio legis unter den Begriff „Schlüsselqualifikationen“ i.S.v. $\$ 5$ a Abs. 3 S. 1 DRiG nur Fertigkeiten gefasst werden, die aus dem Bereich der Kommunikation stammen und für die Rechtspraxis bedeutsam sind. Bestärkt wird diese Forderung auch von Vertretern aus der Berufspraxis, die aus ihrer Alltagserfahrung heraus unter den Begriff der juristischen Schlüsselqualifikationen insbesondere rhetorische und kommunikative Fertigkeiten fassen. Denn in fast jedem juristischen Beruf sei es nötig, mit Worten zu überzeugen oder zu schlichten, durch Fragetechnik an Informationen zu gelangen oder einen komplexen Sachverhalt laienverständlich zu erklären.

Weiter bemängelt das Symposium, dass bei den Angeboten der Fakultäten keine einheitlichen Standards bestehen - insbesondere was den zeitlichen und inhaltlichen Umfang anbelangt: Das Spektrum reicht von halbtägigen Vorlesungsformaten bis hin zu mehrsemestrigen Mediationsausbildungen.

\section{Schlüsselqualifikationen benötigen besondere Lehr- und Lernformate}

Da es bei Schlüsselqualifikationen mehr um das Erlernen von Fertigkeiten als um die Vermittlung reinen Sachwissens geht, sind alternative Lehr- und Lernformate zur klassischen Vorlesung nötig. Das Symposium erachtet daher nur solche Formate als sinnvoll, die insbesondere diese Kriterien erfüllen:

- Individualität und persönliches Feedback

- Praxisbezogenheit

- Nachhaltigkeit, insbesondere durch aktive Mitwirkung und Übung.

3 Verhandlungsmanagement, Gesprächsführung, Rhetorik, Streitschlichtung, Mediation, Vernehmungslehre und Kommunikationsfähigkeit.

4 Eine Übersicht zu den gesetzlichen Berücksichtigungsformen in den Ausbildungsgesetzen und -ordnungen der Bundesländer unter http://www.uni-regensburg.de/rechtswissenschaft/fakultaet/regina/sq/index.html (8.4.2014). 
Als geeignete Formate werden beispielhaft genannt: Moot Court, Law Clinic, Redewettstreit, Projektstudium, Workshop und die Integration in wissenschaftliche Seminare.

Tendenziell sind im Bereich der Schlüsselqualifikationen Blockveranstaltungen gegenüber semesterbegleitenden, zweistündigen Kursen vorzuziehen. Letztere mögen bessere Voraussetzungen für einen nachhaltigeren Lernerfolg bieten. In Blockveranstaltungen sind jedoch die zum Kompetenzerwerb erforderlichen Übungen besser als in zweistündigen Einheiten zu realisieren. Schließlich sprechen für diese auch der enge Semesterwochenplan der Studenten sowie die zumeist angespannte Raumsituation der Universitäten.

Grundsätzlich sollen Schlüsselqualifikationen i.S.v. $\$ 5$ a Abs. 3 S. 1 DRiG nur in Formaten angeboten werden, die sich ausschließlich an Jurastudenten richten. Gemeinsame Angebote mit Studenten anderer Fachrichtungen sind jedoch beispielsweise bei fakultätsübergreifenden Projekten denkbar.

\section{Schlüsselqualifikationsveranstaltungen sollen obligatorisch sein}

Verpflichtend im Studienverlauf sollte mindestens eine frei wählbare Veranstaltung sein. Fakultative Angebote stärken zwar die Eigenverantwortung und werden überwiegend von motivierten Teilnehmern besucht. Verpflichtende Kurse aber können bei nicht intrinsisch motivierten und/oder schüchternen Studenten das Bewusstsein für den Nutzen wecken und sie ermutigen, versteckte Fähigkeiten zu entdecken.

\section{Keine Noten in Kursen, aber Berücksichtigung in Prüfungsergebnissen}

Dozenten sollen in Lehrangeboten zu Schlüsselqualifikationen den Studenten anstelle von Noten individuelle Rückmeldungen zu Fertigkeiten und Entwicklungsfeldern geben. Basis einer Note müsste nämlich eine Klausur oder eine praktische Leistung (z. B. ein Vortrag) sein. Eine Klausur setzt eine umfangreiche Vermittlung theoretischen Wissens voraus, welche nicht das Ziel im kompetenzorientierten Bereich der Schlüsselqualifikationen sein sollte. Bei praktischen Leistungen führt eine Benotung zu einem „Prüfungsverhalten“ durch bloßes Erfüllen des vermeintlich Erforderlichen. Gehemmt wird so aber ein Ausprobieren, das mit individuellem Feedback und konstruktiver Kritik beantwortet werden kann. Auch wirkt sich eine Benotung der Übungen bei schwächeren, aber verbesserungswilligen Studenten negativ auf deren Motivation aus.

In juristischen Prüfungen sollen jedoch gem. $\mathbb{S}$ d Abs. 1 S. 1 DRiG Schlüsselqualifikationen berücksichtigt werden. Dementsprechend sollen sich hier diese Fertigkeiten (insbesondere Rhetorik und Kommunikationsfähigkeit) auch auf die Prüfungsnote auswirken. Das Symposium diskutiert, ob zwei getrennte Teilnoten (Schlüsselqualifikationen/Inhalt) ausgewiesen werden oder Schlüsselqualifikationen einen Aspekt bei der Bildung einer Gesamtnote ausmachen sollen. 


\section{Sowohl Juristen als auch Nicht-Juristen als Dozenten}

Geeignete Dozenten sind nach den Erfordernissen der jeweiligen Schlüsselqualifikation auszuwählen. Entscheidend soll dabei weniger die formale Qualifikation sein als mehr die individuellen didaktischen und inhaltlichen Fertigkeiten. Während für Nicht-Juristen ihr Fachwissen spricht, fällt Juristen die anwendungs- und fallbezogene Vermittlung leichter.

Wünschenswert sind daher Nicht-Juristen mit Bezug zur juristischen Praxis und insbesondere Juristen mit zusätzlicher Trainerausbildung oder anderer Doppelausbildung sowie Tandems aus juristischen und nicht juristischen Dozenten.

\section{Studentische Tutoren}

Tutoren können einerseits eigenständige Lehr- und Lernformate zu Schlüsselqualifikationen anbieten, andererseits Kleingruppen leiten. In den Kleingruppen sind Falllösungen und Referatsrückmeldungen genauso denkbar wie simulierte Mandantengespräche, die sich an Patientengesprächssimulationen aus dem Medizinstudium anlehnen.

Studenten als Dozenten können durch die Nähe zu ihren Kommilitonen die Motivation steigern und eine positive Lernatmosphäre begünstigen. So steht das Lernen aus Fehlern im Vordergrund, nicht die Angst vor Fehlern. Das kompetitive Denken in Lerngruppen kann anregend wirken und zu einer Selbstkontrolle führen, die eine externe Kontrolle entbehrlich macht. Sind zur Anleitung des Kompetenzerwerbs jedoch (Praxis-)Erfahrung oder besondere Kenntnisse nötig, scheiden Tutoren auch aufgrund mangelnder Akzeptanz durch ihre Kommilitonen aus.

Um Tutoren entsprechend anzuleiten, sind didaktisch geschulte Dozenten als Multiplikatoren erforderlich. Den Tutoren selbst bietet das „Lernen durch Lehren“ wiederum die Gelegenheit zum Kompetenzerwerb.

\section{Besondere Auswahl und Betreuung von Dozenten}

Bei der Auswahl geeigneter Dozenten sollen die fachlich zuständigen Lehrstühle zur Qualitätssicherung eingebunden werden. Hilfreich sind insbesondere auch klare und standardisierte Auswahlkriterien und -mechanismen.

Dozenten schätzen neben der organisatorischen Begleitung am Veranstaltungstag insbesondere wechselseitige Evaluationen, Weiterbildungsmöglichkeiten, die Gelegenheit zum Austausch mit anderen Dozenten und einen klaren Ansprechpartner. Um dies alles zu gewährleisten, ist gegenüber einer Betreuung durch einzelne Lehrstühle eine Betreuung durch eine zentrale Stelle der Universität oder der Fakultät vorzugswürdig. 